\title{
Implementación del consejo breve para la reducción del uso de alcohol y tabaco en el primer nivel de atención: un estudio cualitativo
}

\author{
Verónica Lozano Morales (Di,2,3, Norberto Francisco Hernández-Llanes (D44, Billy Raúl Millán Hernández ${ }^{5}$, \\ Rafael Antonio Serna-Arreguin ${ }^{4}$, Leonor Villafranca Salgado', Juan Arturo Sabines Torres ${ }^{4}$, Gady Zabicky Sirot ${ }^{4,6}$ \\ IJurisdicción Sanitaria Núm. 4, Instituto de Salud del Estado de México (ISEM), Tenango del Valle, Estado de México \\ ¿Universidad Tecnológica de México (UNITEC), Campus Toluca, Estado de México \\ ${ }^{3}$ Facultad de Medicina, Universidad de la Salud (UNSA), Toluca, Estado de México \\ ${ }^{4}$ Comisión Nacional contra las Adicciones (CONADIC), Secretaría de Salud, Ciudad de México \\ ${ }^{5}$ Subdirección de Prevención y Control de Enfermedades, Instituto de Salud del Estado de México (ISEM), Metepec, Estado de México. \\ 'Instituto Nacional de Psiquiatría "Ramón de la Fuente Muñiz" (INPRFM), Secretaría de Salud, Ciudad de México.
}

\section{RESUMEN}

Introducción: en México el consumo de tabaco y alcohol representa un problema de salud pública. Modelos de tamizaje, intervención breve y referencia a tratamiento han mostrado eficacia en disminuir dicho consumo, por lo que se emplean en distintos escenarios. Una de sus características más importantes es la cercanía de los profesionales con las situaciones y problemáticas sobre el consumo. Pese a la amplia experiencia en el desarrollo de estos modelos, han sido poco adaptados para su uso en personal médico y de salud en el primer nivel de atención. Objetivo: describir barreras y oportunidades encontradas durante la implementación de un proyecto piloto de tamizaje, consejería breve y referencia a tratamiento dirigido al personal médico y afín del primer nivel de atención en salud. Método: se realizaron dos grupos focales en una muestra aleatorizada de 15 profesionales de salud certificados de un total de 64, que desarrollaron experiencia práctica realizando intervenciones con la población de su establecimiento de salud. Resultados: se encontraron diversas barreras de comunicación, personales y organizacionales; sin embargo, también se identificaron oportunidades que emergen de la implementación de este modelo. Discusión y conclusiones: el uso de intervenciones breves en el primer nivel de atención tiene ventajas para quien las aplica y las recibe, como el incremento en la productividad de las acciones de tamizaje y consejería breve reportadas en el Sistema de Información en Salud, así como la oportunidad de brindar al usuario una intervención personalizada enfocada específicamente en los problemas de la población local.

Palabras clave: consejo breve, uso de alcohol, uso de tabaco, atención primaria en salud, grupos focales.

\begin{abstract}
Introduction: in Mexico, tobacco and alcohol consumption represent a public health problem. Screening, brief intervention, and treatment models had shown effectiveness to reduce substances use, which is why they have been widely used in a variety of settings. The proximity of trained human resources with consumption problems and diverse situations is one of the most important opportunities they offer. Despite the extensive experience in developing these models, they have been poorly adapted for use by medical and health personnel at the first level of care. Objective: describe barriers and opportunities encountered during the operational implementation of a pilot project for screening, brief counseling and treatment aimed at medical personnel and related to the first level of health care in a Sanitary Jurisdiction of the State of Mexico. Method: two focus groups were carried out on a randomized sample of 15 of 64 certified health professionals who developed practical experience performing interventions with the population of their Health Establishment. Results: communication, personal and organizational barriers were found, however, opportunities that emerged from the implementation of this intervention model were also identified. Discussion and conclusions: the implementation of these interventions in the first level of care has various advantages for the personnel who apply them and the population that receives it, among which the increase in productivity of the screening and brief counseling actions reported in the System of Health Information as well as the opportunity to provide the user a personalized intervention specifically focused on their local population-based problems.
\end{abstract}

Keywords: brief counseling, alcohol drinking, tobacco use, primary health care, focus group.

\section{Autor de correspondencia:}

Norberto Francisco Hernández Llanes. Tel.: 5550621600 ext. 59232. Correo electrónico: norberto.Ilanes@salud.gob.mx

Recibido: 25 de marzo de $2020 \quad$ Aceptado: 07 de mayo de 2020 doi: 10.28931/riiad.2020.1.03 


\section{INTRODUCCIÓN}

En México, el consumo de alcohol y tabaco representa un importante problema de salud pública, debido al uso generalizado de estas sustancias por parte de la población. El alcohol es la principal sustancia adictiva consumida en nuestro país, ya que de acuerdo con la Encuesta Nacional de Consumo de Drogas, Alcohol y Tabaco (ENCODAT, 2016-2017; Villatoro-Velázquez et al. 2017), en población de 12 a 65 años, la prevalencia nacional del consumo de alcohol alguna vez en la vida fue de $71 \%$ (del cual $62.6 \%$ corresponde a mujeres y $80.1 \%$ a hombres), el consumo de alcohol en el último mes fue de $35.9 \%$ ( $24.4 \%$ mujeres y $48.1 \%$ los hombres), y el consumo diario fue de $2.9 \%$ (mujer $1.4 \%$ y hombre $4.5 \%$ ). El tabaco, por su parte, es la segunda sustancia psicoactiva más consumida en nuestro país y durante 2016 el porcentaje de fumadores actuales ${ }^{1}$ fue $17.6 \%$ ( $8.7 \%$ mujeres y $27.1 \%$ en hombres), mientras que el porcentaje de fumadores diarios fue de $6.4 \%$, con $3.1 \%$ mujeres y 10.0\% hombres (ENCODAT, 2016-2017; Reynales-Shigematsu et al. 2017).

Además del uso extendido de estas sustancias, también representan un problema importante de salud pública, ya que ambas representan dos de los 10 principales factores de riesgo modificables a la salud por su asociación con enfermedades no transmisibles, son responsables del $11.6 \%$ del total de años de vida saludables perdidos (AVISAS), que equivalen a 2,954.29 AVISAS por cada 100,000 habitantes (GBD 2017 Risk Factor Collaborators, 2018).

Ante este panorama, la Organización Mundial de la Salud (OMS, 2017) ha recomendado las intervenciones breves como medidas costo-efectivas para ofrecer intervenciones individuales basadas en evidencia científica a personas con diferentes patrones de consumo (consumidores ocasionales, consumidores excesivos, personas con abuso) para disminuir o eliminar el consumo de alcohol y tabaco.

Los modelos de tamizaje, intervención breve y referencia a tratamiento (SBIRT, por sus siglas en inglés) surgieron en la década de los 80 para brindar alternativas de atención a los daños potenciales causados por el uso de sustancias en todo el espectro de consumo (Bray, Del Boca, McRee, Hayashi, \& Babor, 2017).

Este modelo es un enfoque comprensivo, integrado y de salud pública para brindar servicios de intervención temprana y tratamiento dirigido a personas con trastor- nos por uso de sustancias, así como para quienes están en riesgo de desarrollar estos desórdenes (SAMHSA, 2011; Babor, Del Boca, \& Bray, 2017 ). Involucra una fase de tamizaje que busca identificar tanto el patrón de consumo de la persona atendida (cantidad, duración y frecuencia), como los problemas de salud ocasionados, seguida por la retroalimentación del resultado obtenido en el tamizaje. Posteriormente, se realiza la fase de intervención breve, cuyo objetivo es que los consumidores identifiquen los riesgos o eventos adversos de su consumo y exploren alternativas para reducirlo. Finalmente, se lleva a cabo la referencia al tratamiento, que tiene como finalidad enviar a los usuarios con problemas de consumo a un servicio especializado de atención de las adicciones (Beyer, Lynch, \& Kaner, 2018; Cowell, Dowd, Mills, Hinde, \& Bray, 2017).

Diversos estudios han demostrado que las intervenciones SBIRT son efectivas para disminuir el consumo excesivo de alcohol (US Preventive Services Task Force, 2018). Sin embargo, para su implementación se debe considerar que existen diversas barreras que afectan el éxito en la aplicación. Johnson, Jackson, Guillaume, Meier y Goyder (2011) encontraron barreras generales para la implementación, como falta de recursos y de entrenamiento a los profesionales, apoyo por parte de las autoridades y carga elevada de trabajo. Por su parte, Aalto, Pekuri y Seppa (2003) refirieron como barreras para la implementación la presencia de problemas operativos durante la ejecución de las intervenciones, percepción de menor autoeficacia de los profesionales de salud, falta de lineamientos para aplicar el consejo breve, dificultad para identificar bebedores problemáticos en etapas tempranas y problemas para iniciar la discusión con los pacientes. D'Souza-Li y Harris (2016) identificaron otras barreras, como la falta de validez y confiabilidad del cuestionario de tamizaje, el uso inadecuado de la entrevista motivacional, el entrenamiento insuficiente a los profesionales de salud, la falta de tiempo y la carencia percibida de servicios especializados para la referencia de los usuarios, que afectan la efectividad de estas intervenciones. D'Onofrio, Pantalon, Degutis, Fiellin y O'Connor (2005) describieron algunas barreras por parte de los usuarios durante la entrevista motivacional, como la negativa a discutir su propio consumo de alcohol, negativa a autoidentificarse como un consumidor problemático durante la intervención, o autoeficacia disminuida para cambiar los patrones de consumo y con ello disminuir su riesgo en salud. Otras barreras

\footnotetext{
${ }^{1}$ La prevalencia de fumadores actuales se define en la ENCODAT 2016-2017 como el porcentaje de la población que actualmente fuma tabaco, enmarcado en un periodo de 30 días.
} 
individuales que han sido encontradas son la dificultad en cambiar la práctica del modelo actual, resistencia y críticas de los colegas y falta de confianza en la evidencia (Spallek et al., 2010).

Adicionalmente, las habilidades técnicas e interpersonales de los profesionales de la salud son variables importantes en el proceso y resultado de la intervención, particularmente cuando se contempla a personal no especializado en intervenciones conductuales, como médicos, enfermeras, trabajadores sociales $u$ otro personal de salud. Se han descrito diferencias en la implementación de intervenciones SBIRT, según los profesionales hacia los cuales va dirigido (Wamsley, Satterfield, Curtis, Lundgren, \& Satre, 2018), lo que resalta la necesidad de una adecuada capacitación del personal (Ordean, Forte, Selby, \& Grennell, 2019), así como el apoyo de la institución donde laboran (Anderson et al., 2003), y el liderazgo y compromiso de las autoridades (Babor et al., 2017; van der Westhuizen et al., 2019).

México tiene una larga tradición en el desarrollo de modelos SBIRT para el consumo de alcohol y otras sustancias (Tiburcio-Sainz \& Natera-Rey, 2013), los cuales se han implementado con éxito en diversos escenarios y con distintas poblaciones (Martínez, Jiménez Pérez, Félix Romero, \& Morales Chainé, 2018; Salazar, Pérez Castro, Ávila Hernández, \& Vacio, 2012; Salazar, Valdez Ruíz, Martínez, \& Pedroza, 2010; Tiburcio, Lara, Martínez, Fernández, \& Aguilar, 2018). Sin embargo, pocos trabajos han evaluado la implementación de intervenciones SBIRT realizadas por personal médico y de salud adscrito al primer nivel de atención en el país. La identificación de las barreras y oportunidades en este contexto permitirá mejorar la forma en la que los modelos de intervención son transferidos hacia las distintas instituciones de salud, por lo que el presente trabajo busca describir las barreras y oportunidades encontradas durante la implementación operativa de un proyecto piloto de tamizaje, consejería breve y referencia a tratamiento, a través de grupos focales conformados por personal de la rama médica y paramédica de primer nivel de atención capacitados y certificados en un Estándar de Competencias de consejería breve, adscritos a unidades médicas de primer nivel en una Jurisdicción Sanitaria del Estado de México.

\section{MÉTODO}

\section{Diseño del estudio}

Se trata de un estudio cualitativo, transversal y descriptivo, realizado a través de la implementación de dos grupos focales dirigidos a personal médico y de salud capacitado y certificado, adscrito a los servicios de primer nivel de atención en la Jurisdicción Sanitaria núm.
4 "Tenango del Valle" del Instituto de Salud del Estado de México.

La descripción de la implementación operativa del proyecto piloto de capacitación y certificación se detalla en el Anexo 1.

\section{Participantes}

Los grupos focales se integraron por siete y ocho personas, respectivamente $(n=15)$. La selección de los participantes de los grupos focales se hizo por muestreo aleatorio simple, tomando como universo de participantes el total de personas capacitadas y certificadas en el Estándar de Competencias EC-0897 (CONOCER, 2017) de la Jurisdicción $(N=64)$. Dicha estrategia permite que los participantes de los grupos focales sean representativos del total de personas capacitadas y certificadas en la Jurisdicción.

\section{Procedimiento}

Para el reclutamiento, a los participantes seleccionados se les envió por correo electrónico una carta-invitación en la cual se les explicó el objetivo y duración del grupo focal, las características de su participación, así como sus derechos y obligaciones durante el proceso.

Los grupos focales se realizaron en el Salón de Usos Múltiples del Centro de Salud de Santa María Jajalpa, en el municipio de Tenango del Valle, Estado de México, durante la jornada laboral (Grupo 1 a las 9:30 hrs. y Grupo 2 a las 12:30 hrs.). El día del estudio se dio la bienvenida a los participantes y se impartió una plática informativa, donde nuevamente se les explicó el proceso, se enfatizó el carácter voluntario de la misma y se les pidió que consintieran por escrito su participación.

Después los participantes requisitaron un cuestionario de datos sociodemográficos y se realizaron los grupos focales. Dos psicólogos especialistas fungieron como moderador y observador en el grupo focal, respectivamente. Se diseñó una guía de entrevista que se utilizó durante el grupo focal como apoyo para iniciar la discusión respecto a los temas de interés. Los grupos focales tuvieron una duración de una hora con 30 minutos.

\section{Consideraciones éticas}

El presente estudio se realizó con estricto apego al Reglamento de la Ley General de Salud en Materia de Investigación en Salud, con relación a los Aspectos Éticos de la Investigación en Seres Humanos y sobre la Investigación en Grupos subordinados. Cada participante leyó y firmó por duplicado un consentimiento con información 
sobre el objetivo y duración del grupo focal, las características de su participación, sus derechos y obligaciones, el nombre y los datos de contacto del responsable del estudio. Se solicitó la firma de dos testigos para asegurar que la participación fuera voluntaria, se entregó un ejemplar al participante y un ejemplar quedó a resguardo de los investigadores, y también consintieron por escrito la grabación en audio de su participación.

Para asegurar la confidencialidad de las respuestas, se les asignó un número consecutivo para la identificación del Cuestionario de Datos Sociodemográficos y durante la grabación de audio en grupos focales no se utilizó ningún identificador por parte de los participantes.

\section{Análisis de datos}

La información obtenida del Cuestionario de Datos Sociodemográficos fue codificada y capturada en Microsoft Excel, donde se analizaron las frecuencias por cada variable y se calcularon estadísticos descriptivos para éstas (media y desviación estándar para variables escalares y proporción para variables nominales y ordinales).

Al mismo tiempo, se transcribieron las grabaciones de audio de los grupos focales para posteriormente ser analizadas en categorías semánticas, según la metodología propuesta por Barbour (2014). Las categorías semánticas principales utilizadas para el análisis fueron barreras y oportunidades percibidas, y se analizaron a través de las cuatro etapas de la implementación del proyecto piloto: convocatoria, capacitación, certificación y ejecución. El análisis se realizó con el software MAXQDA versión 20.0.7 (Software, 2019).

\section{RESULTADOS}

Para la presentación de los resultados, se exponen primero las características sociodemográficas de los participantes, para después mostrar los resultados del análisis de categorías de los grupos focales.

\section{Características sociodemográficas}

Al comparar las características sociodemográficas de los participantes de los grupos focales, se encontraron varias similitudes con respecto al total de personas capacitadas de la Jurisdicción, lo cual sugiere que la muestra estudiada es representativa. Esto se puede observar en la Tabla 1.

Tabla 1

Comparación de las características generales de muestra que participó en los grupos focales con respecto al personal certificado

\begin{tabular}{|c|c|c|}
\hline & $\begin{array}{c}\text { Participantes } \\
\text { (Grupos focales) } \\
(n=14)\end{array}$ & $\begin{array}{c}\text { Referencia } \\
\text { (Personal certificado) } \\
(n=50)\end{array}$ \\
\hline \multicolumn{3}{|l|}{ Sexo $(\%)$} \\
\hline Mujer & 78.6 & 76.0 \\
\hline Hombre & 21.4 & 24.0 \\
\hline \multicolumn{3}{|l|}{ Nivel escolar (\%) } \\
\hline Bachillerato/Técnico & 21.4 & 20 \\
\hline Licenciatura & 64.3 & 66 \\
\hline Especialidad & 0 & 8 \\
\hline Maestría & 14.3 & 6 \\
\hline \multicolumn{3}{|l|}{ Área de especialidad (\%) } \\
\hline Psicología & 28.6 & 22 \\
\hline Medicina & 28.6 & 18 \\
\hline Enfermería & 21.4 & 20 \\
\hline
\end{tabular}


Comparación de las características generales de muestra que participó en los grupos focales con respecto al personal certificado (continuación)

\begin{tabular}{lcc}
\hline & $\begin{array}{c}\text { Participantes } \\
\text { Grupos focales }) \\
(n=14)\end{array}$ & $\begin{array}{c}\text { Referencia } \\
\text { Personal certificado }) \\
(n=50)\end{array}$ \\
\hline Otras áreas de salud & 14.3 & 14 \\
Otras áreas no relacionadas con salud & 7.1 & 20 \\
Sin especialidad & 0 & 6 \\
Estatus de escolaridad (\%) & 100 & 88 \\
Concluida & 0 & 22 \\
Trunca & & 22 \\
Evaluadores (\%) & 57.1 & 2 \\
Evaluador 1 & 0 & 22 \\
Evaluador 2 & 21.4 & 12 \\
Evaluador 3 & 7.1 & 20 \\
Evaluador 4 & 14.3 & 14 \\
Evaluador 5 & 0 & 8 \\
Evaluador 6 & 0 & 22 \\
Evaluador 7 & & 2 \\
\hline
\end{tabular}

La edad promedio de los participantes se ubicó en 36.8 años $(D E=10.2)$, sin encontrar diferencias significativas con el total de personas certificadas $(36.5, D E=9.3$ años), $78.6 \%$ fueron mujeres, sin observarse diferencias significativas respecto al total de personas certificadas. Tampoco se encontraron diferencias en los niveles bajos de escolaridad (bachillerato y licenciatura), aunque entre los participantes del grupo focal hubo mayor presencia de personas con grado de maestría. Al analizar el área de especialidad, se observó que las áreas predominantes en el grupo focal fueron Psicología y Medicina (57.2\%) y una menor proporción de personas de áreas no relacionadas con la salud (como administración o antropología), con respecto al total de personas certificadas $(7.1 \%$ vs $20 \%)$.

En cuanto a los evaluadores asignados, se obtuvo una diferencia importante respecto a los participantes del Evaluador 1 (57.1\% del total de los participantes) y la ausencia de participantes del Evaluador 2, 6 y 7 en el grupo focal. Finalmente, no se observaron diferencias en los conocimientos previos de los participantes respecto al total de personas certificadas (promedio de la evaluación diagnóstica 6.5, $D E=2.2$, contra el total de personas certificadas 6.8, $D E=2.0$ ).

\section{Análisis de los grupos focales}

Para facilitar la presentación de los resultados, se utilizará una secuencia que va desde los antecedentes profesionales en el tema de adicciones, convocatoria de participación, capacitación, certificación en consejo breve y ejecución del consejo breve en población. Sin embargo, es necesario resaltar que durante los grupos focales la dinámica de los temas no necesariamente fue lineal, pues se avanzaba y regresaba entre los mismos.

La mayoría de los participantes refirió no tener un antecedente en materia de formación en adicciones; algunos tuvieron capacitaciones organizadas por la Jurisdicción o por el Centro de Atención Primaria en Adicciones (CAPA), pero no se identificó un programa de formación continua o de profesionalización al que tuviera acceso el personal de primer nivel de atención en salud. 
Los participantes afrontaron diversas barreras durante la convocatoria al curso, sobre todo relacionadas con problemas de comunicación y las instrucciones para la inscripción. Esto fue esperable debido a que las actividades prioritarias varían entre los diversos municipios, por lo que la asignación de profesionales a las capacitaciones dependió principalmente de las propias necesidades del lugar. Así, por ejemplo, varios participantes señalaron que la información recibida fue limitada, por ejemplo:

"Mi coordinación nada más me notificó 'Mañana te vas a un curso, dame tus papeles, necesito tu fotografía, tu CURP'. Realmente yo desconocía a qué venía" (Mujer, G1).

También algunos participantes mencionaron una preferencia de convocatoria hacia ciertas áreas profesionales por parte de los superiores, por ejemplo, el área de psicología o de enfermería. Un participante identificó problemas para el envío electrónico de sus documentos.

La capacitación a los profesionales de la salud tuvo comentarios muy positivos, resaltando la naturaleza teórico-práctica asociada a la consejería (ya que al tratarse de una competencia la capacitación en el desempeño de la consejería es fundamental), así como las características profesionales de los instructores:

"Es un curso realmente interesante y productivo para nosotros" (Hombre, G1).

Una situación que surgió del análisis fue que varios participantes expresaron sentirse "ansiosos" o "inquietos", debido a que las fases de Capacitación y la Certificación estuvieron ligadas, es decir, que tomar la Capacitación comprometía al participante a presentar la evaluación para la Certificación. Por el contrario, algunos consideraron esto una ventaja, ya que se sentían más comprometidos por el hecho de certificar una habilidad.

Una dificultad sugerida por varios de ellos fue la rigidez de la secuencia de la intervención SBIRT, como problemas para pasar de un tema a otro durante la consejería y las desviaciones de los usuarios respecto a la información que se les solicita. Por ejemplo:

"Me cuesta mucho trabajo que me digan "A, B, C, D", porque a veces en la plática el tema se puede como desviar, lo regresas al tema y sigues" (Mujer, G1).

A pesar de esto, la mayoría de los participantes concordó en que la capacitación fue útil como preparación para la Evaluación en el Estándar de Competencias. Entre las sugerencias que aportaron para mejorar la
Capacitación y la Evaluación estuvieron el uso de elementos multimedia (como videos y manuales electrónicos) y la práctica supervisada y con menor percepción de utilidad, los manuales impresos y los cursos en línea.

El proceso de Certificación fue calificado por la gran mayoría de los participantes como sumamente estresante. En el análisis se identificaron dos categorías relacionadas con el estrés de la evaluación, que fueron las características y experiencia personal y las expectativas en cuanto al desempeño de los otros. Con respecto a la primera, la confianza en sí mismo y la experiencia personal permitió que los participantes se sintieran confiados y tuvieran mejor desempeño durante la Certificación:

"Obviamente en el proceso de evaluación, pues te pones nervioso, entonces yo creo que al momento de tratar como tratamos al paciente, nos sentimos cómodos: cuestionando al paciente, y conforme vas trabajando con el paciente te vas acordando y vas memorizando lo más importante, por ejemplo, cuantos mililitros tiene una cerveza a diferencia de una copa" (Mujer, G2).

También hubo mención de preferencias de los evaluadores hacia ciertos candidatos. Esto a pesar de que las evaluaciones se realizaron de forma estandarizada (CONOCER, 2010).

Las expectativas respecto al desempeño de los compañeros también fue un tema que resultó de importancia. Aunque las evaluaciones fueron individuales, al parecer los tiempos de espera entre evaluaciones, los comentarios informales entre compañeros y las comparaciones de resultados entre ellos tuvieron efecto en los niveles de estrés de los participantes, lo cual se podría reflejar en su desempeño. Por ejemplo:

\section{"Y yo dije 'la psicóloga no pasó, a mí qué me espera"” (Mujer, G1).}

Los evaluadores, por su parte, cayeron en dos categorías. Algunos fueron reconocidos como comprensivos, ponían atención y estaban orientados a los participantes, y otros fueron reconocidos como estrictos y más orientados a la prueba.

Una situación interesante es que los participantes con especialidad en psicología señalaron haber tenido dificultad para ejecutar la intervención, debido principalmente al sesgo profesional de profundizar sobre la información en la entrevista (como por ejemplo en los motivos, preferencias, consecuencias del consumo, etcétera).

Con respecto a la Ejecución, todos los participantes refirieron haber realizado la intervención en la Unidad de 
Salud en la que están adscritos. Mencionaron también que fue más común la intervención en mujeres que en hombres; sin embargo, mencionaron que los usuarios aceptaron de buena manera la intervención y muy pocos usuarios de servicios de salud se negaron a recibirla. Por ejemplo:

"Con las que están en las salas de expulsión en Tococirugía y ahí empiezo a indagar. Muchas de ellas a lo mejor no consumen, pero su pareja y sus suegros sí, y se les da la consejería" (Mujer, G1).

También se describieron diferencias culturales entre las localidades con respecto a la intervención, por ejemplo:

"Cada localidad tiene sus costumbres, entonces no puedes llegar y ya decirles que no a sus costumbres porque ya no van a regresar, o sea literal ¿Para qué voy [al Centro de Salud] si me van a regañar?" (Mujer, G2).

Varios participantes tuvieron resultados positivos por la aplicación de la intervención; por ejemplo, los usuarios referidos iniciaron tratamiento en el CAPA, otros afirman que los usuarios llevaron a sus familiares para que recibieran la intervención. Uno de los casos de éxito compartido fue el siguiente:

"A un paciente con un diagnóstico de tuberculosis ganglionar le pregunté '¿Usted fuma?' Y me contestó que sí, pero ya que le platicamos acerca de la tuberculosis, de lo que es el padecimiento y ya que le hicimos la intervención de consejería breve, tiempo después le volvimos a preguntar y nos dijo que ya nada más uno a la semana" (Hombre, G2).

Un hallazgo inesperado fue que los propios participantes en la capacitación enfrentaron un cambio de actitud sobre su propio consumo, lo que se reflejó en dejar de consumir o disminuir el patrón de consumo:

"Pues en la fiesta sí digo: 'Nada más tres... la cuarta ya corres un riesgo'" (Mujer, G1).

Finalmente, entre otros emergentes, los participantes conversaron sobre problemas logísticos, como retrasos en los tiempos y documentación con errores, aunque no fue una constante en el proceso; además, los propios participantes asumen estas condiciones como parte del proceso de implementación. También se mencionó la necesidad de sensibilizar a los supe- riores respecto a este modelo de intervención, ya que argumentaron falta de apoyo para asistir a las capacitaciones, falta de espacios para realizar la intervención y problemas para registrar las acciones en el sistema por parte del personal no médico/enfermería/ psicología. Por el contrario, los participantes reconocieron que realizar una ceremonia para entregar los certificados fue una motivación para ellos, y en general consideraron que este tipo de intervenciones los ayudan a mejorar el servicio que brindan a sus usuarios.

\section{DISCUSIÓN Y CONCLUSIONES}

De los resultados anteriormente expuestos, se puede concluir que existe una serie de barreras que se presentaron durante la implementación de una intervención de consejería breve en personal adscrito al primer nivel de atención en salud.

Damschroder et al. (2009) han propuesto un marco unificado, en el que la implementación de intervenciones se compone por cinco dominios principales: las características de la intervención, el ambiente externo (en el cual se realiza la intervención), el ambiente interno (que se refiere al ambiente institucional), características de los individuos involucrados y el proceso de implementación. Durante el proceso de implementación, las principales barreras encontradas fueron del tipo organizacional, como deficiencias en la comunicación y falta de involucramiento de las autoridades, lo cual va en el sentido de la evidencia existente en la literatura publicada (Anderson et al., 2003; Martínez et al., 2018). Aunque el proyecto inicialmente se presentó tanto a las autoridades jurisdiccionales como a los coordinadores municipales, resulta necesario involucrarlos durante todo el proceso de implementación.

Con respecto al dominio de las características de los individuos involucrados, la experiencia previa parece no ser determinante en el resultado de los participantes, mientras que la práctica parece impactar, tanto de forma directa al mejorar las capacidades técnicas e interpersonales del profesional de la salud, como de manera indirecta al disminuir el estrés o ansiedad del participante por el proceso de certificación. Con base en esto, se puede considerar que la capacitación con enfoque de competencias es una oportunidad para implementar intervenciones de consejo breve, ya que como lo mencionan Rey-Gamero y Acosta-Ramírez (2013), el enfoque de competencias laborales permite el desarrollo de intervenciones basadas en conocimientos científicos y favorecen el trabajo en conjunto y las habilidades de comunicación en los equipos de Atención Primaria en Salud. 
Un elemento que sorprendió fue que los participantes refirieron un cambio de actitud ante su propio consumo y el de sus familiares. Morales Chainé, Félix Romero, Palafox Palafox y Vázquez Martínez (2019) señalan que la capacitación a profesionales tuvo un impacto positivo sobre las actitudes propias de la consejería breve, como comunicación y empatía. Esto mismo se ha observado en otros profesionales de la salud, como dentistas (Ntouva, Sanatinia, \& Watt, 2018). Sin embargo, hasta donde se tiene conocimiento, muy pocos estudios han abordado la actitud del profesional respecto a su propio consumo, por lo que se recomienda que futuros estudios indaguen la relación entre ésta y el desempeño del profesional de salud en la aplicación de la consejería breve.

Una barrera importante es que, aunque se busca estandarizar la intervención ante diferentes escenarios, es fundamental considerar como parte de su diseño la inclusión de elementos culturales que hagan las intervenciones más locales y permitan dirigirlas a poblaciones más específicas (Manuel et al., 2015). Se ha señalado que las características raciales impactan la forma en la que el profesional de la salud realiza la consejería breve (Satre, Manuel, Larios, Steiger, \& Satterfield, 2015), por lo que para lograr lo anterior es necesario caracterizar el consumo en estas poblaciones e incluso trabajar con estas comunidades para desarrollar mensajes preventivos y establecer límites de consumo alcanzables para dichas poblaciones.

Finalmente, un tema que es poco abordado pero necesario para mejorar la implementación de una estrategia de consejería breve se relaciona con la productividad del personal de salud. Si se considera que a nivel nacional existe un déficit de profesionales médicos, y los que están en el Sistema Nacional de Salud experimentan una carga laboral considerable (Nigenda et al., 2016), la implementación de una intervención en consejería breve no debe ser percibida como una carga administrativa o laboral. En el sistema de salud de Estados Unidos, donde la productividad está relacionada con el ingreso del trabajador, las tasas bajas de reembolso por servicios médicos y las restricciones administrativas para cobrar las intervenciones son barreras documentadas para la implementación (Johnson \& Seale, 2015). Con respecto a esto, una oportunidad de la implementación del consejo breve fue el registro de las acciones en el Sistema Nacional de Vigilancia Epidemiológica, pero también es necesario resolver los problemas relacionados con el registro de las acciones por parte del personal no médico, para que los sistemas de información en salud reflejen las acciones realizadas y con ello se pueda medir el impacto de estas acciones en la población.

\section{AGRADECIMIENTOS}

Los autores agradecen el apoyo de la doctora Marcela Martinelli Herrera, la licenciada Magali Irais Chavero Hernández, la licenciada Alma Rosa García Blancas, la maestra Maricela Cruz Rivera, la maestra Alma Leticia Mora Gómez, la maestra Rosa María Martínez Espíritu Santo, el licenciado Jorge Cortés Bernal y el licenciado José Antonio León Consuelos, de la Comisión Nacional contra las Adicciones, así como a la licenciada Erika Cynthia Loretto Castillo, Enfer Margarita Galván González, al licenciado Eduardo Monroy García y al doctor Moisés Alejandro Nava Romero, de la Jurisdicción Sanitaria núm. 4, por su apoyo durante la capacitación y certificación. También al licenciado Rafael Cortés Fuentes, por sus comentarios y observaciones al manuscrito. $Y$ el reconocimiento al personal de salud adscrito a las cinco Coordinaciones Municipales, ya que sin su profesionalismo y dedicación este trabajo no hubiera sido posible.

\section{ANEXO}

\section{Descripción de la intervención SBIRT:}

La intervención se realizó en dos fases: la primera se llevó a cabo entre enero y mayo de 2019 y consistió en la capacitación y certificación del personal de primer nivel de atención en salud adscrito a 13 establecimientos de salud (Almoloya del Río, Atizapán, Calimaya, Capulhuac, Chapultepec, Joquicingo, Mexicaltzingo, Rayón, San Antonio la Isla, Tenango del Valle, Texcalayacac, Tianguistengo y Xalatlaco), correspondientes a las cinco Coordinaciones Municipales de Salud (Chapultepec, Xalatlaco, Santa Cruz Atizapán, Tenango del Valle y Texcalyacac), todas en el área de influencia de la Jurisdicción Sanitaria núm. 4 "Tenango del Valle", en el Estado de México

La capacitación tuvo una duración de 10 horas divididas en dos sesiones. En la primera sesión se revisaron los temas relacionados con la epidemiología, mecanismos fisiopatológicos, daños a la salud asociados al consumo de alcohol y tabaco y consejo breve; mientras que en la segunda sesión se abordaron aspectos de entrevista motivacional, el cuestionario de ocho preguntas de tamizaje de la intervención y se realizaron ejercicios prácticos de consejería breve apoyados con viñetas clínicas. La capacitación del Grupo 1 (G1) se realizó en enero y la del Grupo 2 (G2) en abril, ambas en 2019.

La carta descriptiva correspondiente está alineada a la estructura del Estándar de Competencias EC-0897 "Proporcionar consejo breve para la cesación del consumo de alcohol y tabaco" (CONOCER, 2017). 
Tabla 2

Personal capacitado y certificado del primer nivel de atención en salud de las Coordinaciones Municipales adscritas a la Jurisdicción Sanitaria núm. 4, primer semestre 2019

\begin{tabular}{|c|c|c|c|}
\hline & \multicolumn{3}{|c|}{ Personal } \\
\hline & \multirow{2}{*}{$\begin{array}{c}\text { Capacitado } \\
(n=110) \\
n\end{array}$} & \multicolumn{2}{|c|}{$\begin{array}{c}\text { Certificado } \\
\quad(n=64)\end{array}$} \\
\hline & & $n$ & $\%$ del total \\
\hline Jurisdicción Sanitaria núm. 4 & 6 & 4 & 66.7 \\
\hline \multicolumn{4}{|l|}{ Coordinaciones Municipales } \\
\hline Capulhuac & 6 & 5 & 83.4 \\
\hline Chapultepec & 14 & 14 & 100 \\
\hline Santa Cruz Atizapán & 12 & 5 & 41.7 \\
\hline Tenango del Valle & 45 & 23 & 51.1 \\
\hline Texcalyacac & 15 & 6 & 40.0 \\
\hline Xalatlaco & 12 & 7 & 58.3 \\
\hline Promedio & & & 63.1 \\
\hline
\end{tabular}

Posteriormente, las personas capacitadas fueron convocadas para ser evaluadas y poder certificarse en el citado Estándar. La evaluación se realizó de manera individual y consistió en una evaluación de desempeños (práctica) y una de conocimientos (teórica), con una duración promedio de 2.5 horas por persona. Todas las evaluaciones fueron hechas conforme al procedimiento establecido en el Estándar de Competencias EC-0076 "Evaluación de la competencia de candidatos con base en el Estándar de Competencias" de CONOCER (2010) y fueron efectuadas por psicólogos especializados certificados en ambos Estándares. Las evaluaciones del G1 se llevaron a cabo en marzo y del G2 en abril de 2019.

En la segunda fase, el personal que fue certificado realizó la intervención SBIRT en la población usuaria de los servicios de salud de los 13 municipios que integran dicha Jurisdicción. Esta fase comenzó en el mes de marzo para el G1 y en mayo para el G2. Con el objeto de reflejar la productividad del personal en las intervenciones de Consejería Breve, el personal adscrito a las unidades de salud reportó sus acciones en el Sistema Único de Información para la Vigilancia Epidemiológica (SUIVE), bajo el código CIE-10 Z13.3 "Examen de pesquisa especial para trastornos mentales y del comporta- miento" (Organización Panamericana de la Salud, 1995). En el periodo comprendido entre marzo y diciembre de 2019, el personal certificado reportó en dicho sistema 6,080 intervenciones en población general usuaria de los servicios de salud (un promedio de 608 intervenciones mensuales, con un rango de 182 a 1,009 acciones).

\section{REFERENCIAS}

Aalto, M., Pekuri, P., \& Seppa, K. (2003). Obstacles to carrying out brief intervention for heavy drinkers in primary health care: a focus group study. Drug and Alcohol Review, 22(2), 169-173. doi:10.1080/09595230100100606

Anderson, P., Kaner, E., Wutzke, S., Wensing, M., Grol, R., Heather, N., \& Saunders, J. (2003). Attitudes and management of alcohol problems in general practice: descriptive analysis based on findings of a World Health Organization international collaborative survey. Alcohol and Alcoholism, 38(6), 597-601. doi:10.1093/alcalc/agg119

Babor, T. F., Del Boca, F., \& Bray, J. W. (2017). Screening, Brief Intervention and Referral to Treatment: implications of SAMHSA's SBIRT initiative for substance abuse policy and practice. Addiction, 112 (Suppl 2), 110-117. doi:10.1111/add.13675

Barbour, R. S. (2014). Analysing Focus Groups. In U. Flick (Ed.), The SAGE Handbook of Qualitative Data Analysis (pp. 313-326). Londres, Inglaterra: Sage. 
Beyer, F., Lynch, E., \& Kaner, E. (2018). Brief Interventions in Primary Care: an Evidence Overview of Practitioner and Digital Intervention Programmes. Current Addiction Reports, 5(2), 265-273. doi:10.1007/s40429-018-0198-7

Bray, J. W., Del Boca, F. K., McRee, B. G., Hayashi, S. W., \& Babor, T. F. (2017). Screening, Brief Intervention and Referral to Treatment (SBIRT): rationale, program overview and cross-site evaluation. Addiction, 112(2), 3-11. doi:10.1111/add.13676

CONOCER. (2010). Evaluación de la competencia de candidatos con base en el Estandar de Competencias (pp. 12). Ciudad de México, México: CONOCER.

CONOCER. (2017). Proporcionar consejo breve para la cesación del consumo de alcohol y tabaco en el primer nivel de atención en salud (pp. 11). Ciudad de México, México: CONOCER.

Cowell, A. J., Dowd, W. N., Mills, M. J., Hinde, J. M., \& Bray, J. W. (2017). Sustaining SBIRT in the wild: simulating revenues and costs for Screening, Brief Intervention and Referral to Treatment programs. Addiction, 112(Suppl 2), 101-109. doi:10.1111/ add. 13650

D’Onofrio, G., Pantalon, M. V., Degutis, L. C., Fiellin, D. A., \& O'Connor P, G. (2005). Development and implementation of an emergency practitioner-performed brief intervention for hazardous and harmful drinkers in the emergency department. Academic Emergency Medicine, 12(3), 249-256. doi:10.1197/j. aem.2004.10.021

D'Souza-Li, L., \& Harris, S. K. (2016). The future of screening, brief intervention and referral to treatment in adolescent primary care: research directions and dissemination challenges. Current Opinion in Pediatrics, 28(4), 434-440. doi:10.1097/ MOP.0000000000000371

Damschroder, L. J., Aron, D. C., Keith, R. E., Kirsh, S. R., Alexander, J. A., \& Lowery, J. C. (2009). Fostering implementation of health services research findings into practice: a consolidated framework for advancing implementation science. Implementation Science, 4, 50. doi:10.1186/1748-5908-4-50

GBD 2017 Risk Factor Collaborators. (2018). Global, regional, and national comparative risk assessment of 84 behavioural, environmental and occupational, and metabolic risks or clusters of risks for 195 countries and territories, 1990-2017: a systematic analysis for the Global Burden of Disease Study 2017. The Lancet, 392(10159), 1923-1994. doi:10.1016/s0140-6736(18)32225-6

Johnson, J. A., \& Seale, J. P. (2015). Implementing alcohol screening and brief intervention in primary care: identifying barriers, proposing solutions. Addiction Science \& Clinical Practice, 10(S1). doi:10.1186/1940-0640-10-s1-a24

Johnson, M., Jackson, R., Guillaume, L., Meier, P., \& Goyder, E. (2011). Barriers and facilitators to implementing screening and brief intervention for alcohol misuse: a systematic review of qualitative evidence. Journal of Public Health, 33(3), 412-421. doi:10.1093/pubmed/fdq095

Manuel, J. K., Satre, D. D., Tsoh, J., Moreno-John, G., Ramos, J. S., McCance-Katz, E. F., \& Satterfield, J. M. (2015). Adapting Screening, Brief Intervention, and Referral to Treatment for Alcohol and Drugs to Culturally Diverse Clinical Populations. Journal of Addiction Medicine, 9(5), 343-351. doi:10.1097/ADM.0000000000000150

Martínez, K., Jiménez Pérez, A. L., Felix Romero, V., \& Morales Chainé, S. (2018). Impact of a brief intervention programs in clinical practice: Barriers and adaptations. International Journal of Psychological Research, 11(2), 27-34. doi:10.21500/20112084.3485

Morales Chainé, S., Felix Romero, V., Palafox Palafox, G., \& Vázquez Martínez, J. L. (2019). Conocimientos, habilidades y actitudes profesionales para la intervención breve en adicciones. Revista Internacional de Investigación en Adicciones, 5(2), 8-20. doi: 10.28931/riiad.2019.2.02

Nigenda, G., Alcalde-Rabanal, J., González-Robledo, L. M., Serván-Mori, E., García-Saiso, S., \& Lozano, R. (2016). Eficiencia de los recursos humanos en salud: una aproximación a su análisis en México. Salud Pública de México, 58(5), 533-542. doi:10.21149/spm.v58i5.8243

Ntouva, A., Sanatinia, R., \& Watt, R. G. (2018). Evaluation of an alcohol screening and brief advice training programme for NHS general dental practitioners. European Journal of Dental Education, 22(1), 34-39. doi:10.1111/eje.12241

Organización Mundial de la Salud. (2017). Lucha contra las ENT: Mejores inversiones" y otras intervenciones recomendadas para la prevención y el control de las enfermedades no transmisibles. Ginebra, Suiza: OMS.

Organización Panamericana de la Salud. (1995). Clasificación estadística internacional de enfermedades y problemas relacionados con la salud, 10a. revisión. Vol. 1. Washington, D.C.: OPS. Recupareado de: http://ais. paho.org/classifications/Chapters/ pdf/Nolume1.pdf

Ordean, A., Forte, M., Selby, P., \& Grennell, E. (2019). Screening, Brief Intervention, and Referral to Treatment for Prenatal Alcohol Use and Cigarette Smoking: A Survey of Academic and Community Health Care Providers. Journal of Addiction Medicine. doi:10.1097/ADM.0000000000000588

Rey-Gamero, A. C., \& Acosta-Ramírez, N. (2013). El enfoque de competencias para los equipos de Atención Primaria en Salud. Una revisión de literatura. Gerencia y Políticas de Salud, 12(25), 28-39. doi: 10.11144/Javeriana.rgsp12-25.ecpe

Reynales- Shigematsu, L. M., Zavala-Arciniega, L., Paz-Ballesteros, W. C., Gutiérrez-Torres, D. S., García-Buendía J. C., Rodriguez-Andrade, M. A., ... Mendoza-Alvarado, L. (2017) Encuesta Nacional de Consumo de Drogas, Alcohol y Tabaco [ENCODAT] 2016-2017: Reporte de Tabaco. Ciudad de México, México: INPRFM.

Salazar Garza, M., Pérez Castro, J. L., Ávila Hernández, O. P., \& Vacio, Á. (2012). Consejo breve a universitarios que consumen alcohol en exceso: resultados iniciales. Psicología y Salud, 22(2), 247-256. doi: 10.25009/pys.v22i2.549

Salazar Garza, M., Valdez Ruíz, J. F., Martínez, K., \& Pedroza, F. (2010). Intervenciones breves con adolescentes estudiantes rurales que consumen alcohol en exceso. Universitas Psychologica, 10(3), 803-815. doi: 10.11144/Javeriana.upsy10-3.ibae

SAMHSA. (2011). Screening, Brief Intervention and Referral to Treatment (SBIRT) in Behavioral Healthcare. Rockville, MD: SAMHSA. 
Recuperado de: https://www.samhsa.gov/sites/default/files/sbirtwhitepaper_0.pdf

Satre, D. D., Manuel, J. K., Larios, S., Steiger, S., \& Satterfield, J. (2015). Cultural Adaptation of Screening, Brief Intervention and Referral to Treatment Using Motivational Interviewing. Journal of Addiction Medicine, 9(5), 352-357. doi:10.1097/ ADM.0000000000000149

Software, V. (2019). MAXQDA 2020 (Version 20.0.7). Berlin, Alemania: VERBI Software.

Spallek, H., Song, M., Polk, D. E., Bekhuis, T., Frantsve-Hawley, J., \& Aravamudhan, K. (2010). Barriers to implementing evidence-based clinical guidelines: a survey of early adopters. Journal of Evidence Based Dental Practice, 10(4), 195-206. doi:10.1016/j. jebdp.2010.05.013

Tiburcio-Sainz, M., \& Natera-Rey, G. (2013). An overview of alcohol screening and treatment programs in the Mexican health system. Addiction Science \& Clinical Practice, 8(S1). doi:10.1186/19400640-8-s1-a77

Tiburcio, M., Lara, M. A., Martínez, N., Fernández, M., \& Aguilar, A. (2018). Web-Based Intervention to Reduce Substance Abuse and Depression: A Three Arm Randomized Trial in Mexico. Substance Use \& Misuse, 53(13), 2220-2231. doi:10.1080/1082 6084.2018.1467452
US Preventive Services Task Force (2018). Screening and Behavioral Counseling Interventions to Reduce Unhealthy Alcohol Use in Adolescents and Adults: US Preventive Services Task Force Recommendation Statement. JAMA, 320(18), 1899-1909. doi:10.1001/jama.2018.16789

van der Westhuizen, C., Myers, B., Malan, M., Naledi, T., Roelofse, M., Stein, D. J., ... Sorsdahl, K. (2019). Implementation of a screening, brief intervention and referral to treatment programme for risky substance use in South African emergency centres: A mixed methods evaluation study. PLoS One, 14(11), e0224951. doi:10.1371/journal.pone.0224951

Villatoro-Velázquez. J. A., Resendiz -Escobar, E., Mujica-Salazar, A., Bretón-Cirett, M., Cañas-Martínez, V., Soto-Hernández. I., ... Mendoza-Alvarado. L. (2017) Encuesta Nacional de Consumo de Drogas, Alcohol y Tabaco [ENCODAT] 2016-2017: Reporte de Alcohol. Ciudad de México, México: INPRFM

Wamsley, M., Satterfield, J. M., Curtis, A., Lundgren, L., \& Satre, D. D. (2018). Alcohol and Drug Screening, Brief Intervention, and Referral to Treatment (SBIRT) Training and Implementation: Perspectives from 4 Health Professions. Journal of Addiction Medicine, 12(4), 262-272. doi:10.1097/ADM.0000000000000410 